\title{
Lapurdum
}

Euskal ikerketen aldizkaria | Revue d'études basques |

Revista de estudios vascos | Basque studies review

$20 \mid 2017$

Numéro $X X$

\section{U ghjattumammò et Barbarone parmi les croquemitaines de la littérature orale corse}

Poli Muriel

\section{OpenEdition}

\section{Journals}

Édition électronique

URL : https://journals.openedition.org/lapurdum/3533

DOI : 10.4000/lapurdum.3533

ISSN : 1965-0655

Éditeur

IKER

Édition imprimée

Date de publication : 1 janvier 2017

Pagination : 169-182

ISBN : 978-2-95534-135-3

ISSN : $1273-3830$

Référence électronique

Poli Muriel, «U ghjattumammò et Barbarone parmi les croquemitaines de la littérature orale corse », Lapurdum [En ligne], 20 | 2017, mis en ligne le 01 janvier 2021, consulté le 30 mars 2022. URL : http:// journals.openedition.org/lapurdum/3533; DOI : https://doi.org/10.4000/lapurdum.3533 


\section{U ghjattumammò et Barbarone parmi les croquemitaines de la littérature orale corse}

Poli MURIEL

BDLC - UMR LISA

Université de Corse

En Corse, comme ailleurs, la transmission intergénérationnelle des légendes et des contes est indissociable de la pratique d'a veghja. Lors de ces veillées, le conteur exploite ses talents oratoires alternant histoires facétieuses et récits terrifiants peuplés de personnages fantastiques.

De telles coutumes n'ont plus cours depuis une soixantaine d'années mais il est toujours possible de glaner, çà et là, des ethnotextes qui mis bout à bout, constituent un corpus oral relativement riche.

Un premier examen des données de terrain toute récentes dessine les contours de la situation d'oralité et plus généralement l'histoire de la communauté étudiée des années 30 à 60.

Sur le plan diégétique, un motif nous interpelle : l'intervention du croquemitaine. À la lecture du corpus, il apparaît en effet comme une figure récurrente.

Ainsi, nous considèrerons les conditions de son intervention supposée et son apparence à la lumière des descriptions révélées par nos enquêtes, confrontées aux développements consacrés aux mythes, proposés par les plus grands folkloristes.

Notre tentative de caractérisation d'un acteur familier aux sociétés de tradition orale nous permet de poser les jalons d'une étude comparatiste plus aboutie.

\section{Localisation et contexte}

\subsection{Cadre de l'étude}

Létude a été commandée par le Pays de Balagne, groupement de communes dont le périmètre correspond à la microrégion du même nom. Elle est exécutée par nos soins ${ }^{1}$, sous la

1. Muriel Poli, MCF $73^{e}$ section CNU, responsable scientifique de l'étude \& Agnès Rogliano-Desideri, docteur en Littérature générale et comparée, 10e section CNU. 
forme d'un contrat de prestation de service encadré par l'UMR LISA de l'université de Corse et le CNRS.

Le projet s'inscrit dans un axe plébiscité par les solliciteurs, désireux de « bâtir une économie du tourisme patrimonial » en valorisant les ressources locales de façon à proposer une offre touristique offre touristique nouvelle. Les acteurs locaux y sont associés à travers l'héritage culturel dont ils sont témoins. La réalisation d'un recueil de contes et légendes de Balagne est soutenue par le Plan de Développement Rural de la Corse et financée par le programme européen « Leader ».

Les témoignages oraux ont été rassemblés entre septembre 2014 et avril 2015 sur un territoire situé au Nord Ouest de la Corse. Il couvre 36 communes et occupe une surface de $957 \mathrm{~km} 2$. La population avoisine 21400 habitants d'après les données de l' « Atlas des territoires de projet » édité en 2009 par la Collectivité Territoriale de Corse.

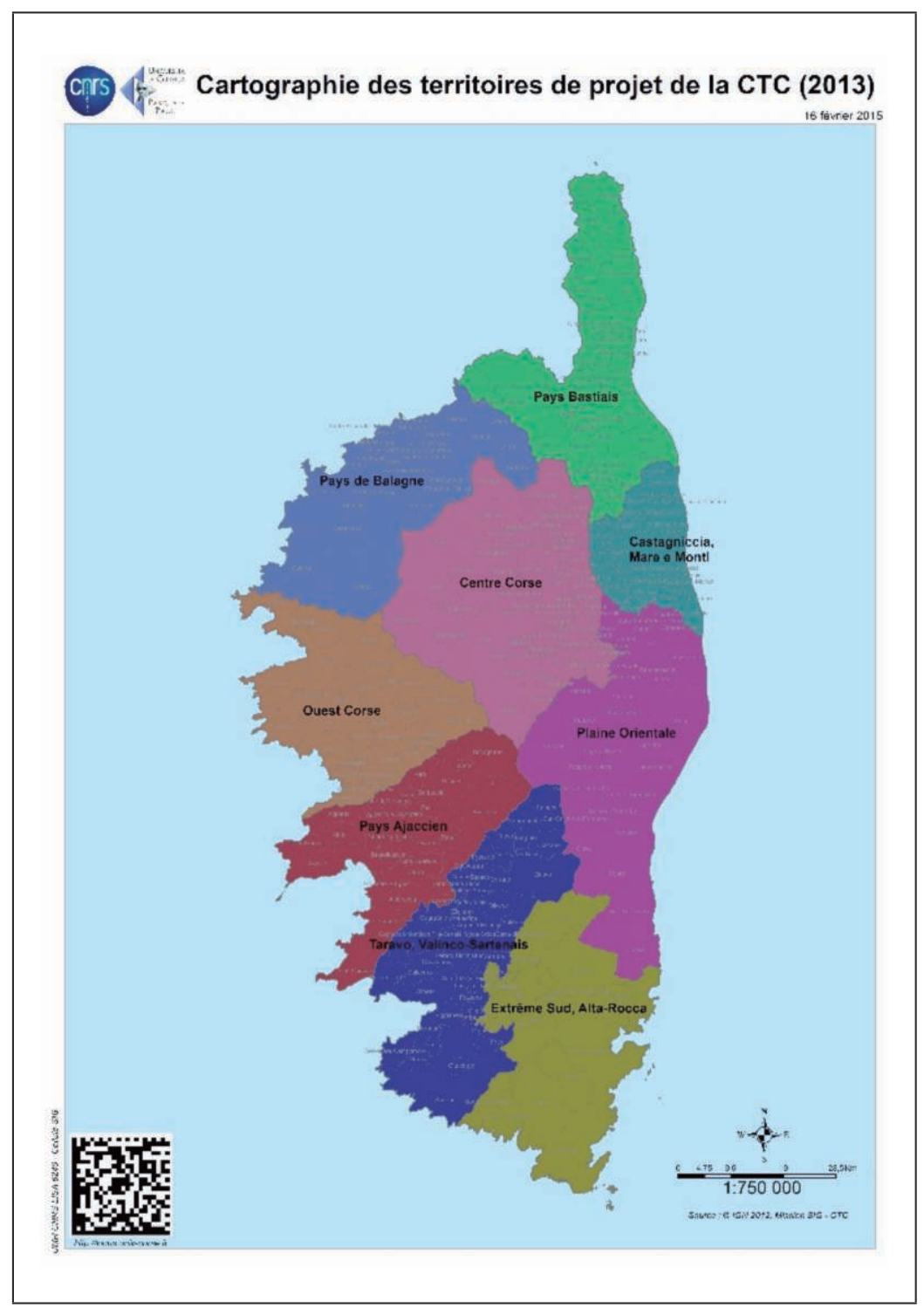

Figure 1 : extrait de la plateforme géomatique LOCUS http:// locus.univ-corse.fr 
Nos prospections s'avèrent inédites si l'on considère la majorité des recherches menées aujourd'hui en Corse sur la littérature orale, puisque quasiment toutes répondent à une démarche bibliographique. Rares sont les chercheurs prêts à traiter le sujet à sa source, réticence probablement dûe à la fois à la pénurie d'informateurs et au caractère chronophage de ce type d'approche...

\subsection{Méthode}

Dans notre approche du territoire, sur lequel nous avons déjà souvent enquêté ${ }^{2}$, nous nous sommes appuyée sur des relais locaux, vecteurs de dynamisme dans des zones désertées au profit des agglomérations littorales. Ainsi, les mairies, le réseau des foyers ruraux et des confréries religieuses nous ont orientée au moment de la recherche de témoins. Nous avons ainsi pu respecter le cahier des charges et rendre compte des 36 communes.

Les personnes ressources nous ont été indiquées comme étant les garants de la société traditionnelle. Le plus âgé est né en 1912 et les plus jeunes en 1953. Ils s'expriment ordinairement en langue corse.

Nous avons utilisé l'entretien comme dispositif exclusif de recueil de données, essentiellement sous la forme semi-directive.

L'emploi de questions ouvertes nous a permis de reconstituer a veghja. Plus précisément: Qui contait ? Où cela se passait-il et quand? Pour quel public? Quels types de récits?

Néanmoins, les textes rapportés forment un ensemble hétéroclite. Les contes et histoires désignés en corse par le terme fola, du latin FABULA, souvent qualifiés de fantastiques, représentent un peu plus de soixante pourcent du corpus ; le reste étant constitué de chroniques paysannes.

Ces dernières n'en sont pas moins révélatrices du mode de vie des balanins (et des corses) dans la première partie du XXe siècle et avant puisque nos informateurs relatent ce que leurs parents ou grands-parents leur ont transmis. Il y est fait mention des activités économiques, du rapport à la religion, à la justice (collusions avec les bandits) ou encore de pratiques magico-religieuses, à la frontière du réel et du fantastique.

Les histoires merveilleuses ont subi — comme le reste de la tradition orale — les ravages du temps et plus encore la dislocation de la société traditionnelle. Leur caractère chimérique n'a pas bien résisté à l'apparition brutale et massive des moyens de communication moderne et la rupture dans la transmission intergénérationnelle de la langue corse a précipité leur déclin.

Malgré tout, à la lecture du corpus, nous voyons se dessiner les traits de ces personnages

2. Différentes prospections destinées majoritairement à alimenter la Banque de Données Langue Corse de l'université de Corse. Mais aussi, l'étude en cours : «Quels littoraux pour demain ?» financée par la Fondation de France, relative au rôle des sentiers dans la dynamique naturelle et culturelle du littoral balanin, sous la responsabilité d'Hélène Melin (MCF Sociologie-Anthropologie de l'université de Lille); nous y traitons le volet ethnolinguistique à travers les savoirs naturalistes traditionnels. 
étonnants. Celui de la vieille maléfique et plus précisément les figures corses du croquemitaine, ont retenu notre attention. Le « monstre imaginaire, fantastique et effrayant, de certains contes de fées, qu'on évoque pour faire peur aux enfants et dont on les menace pour s'en faire obéir » — tel que le définit le CNRTL — s'impose dans notre recueil. Il semble avoir particulièrement marqué les mentalités si l'on considère la réaction de certains de nos informateurs, aujourd'hui encore troublés à son évocation.

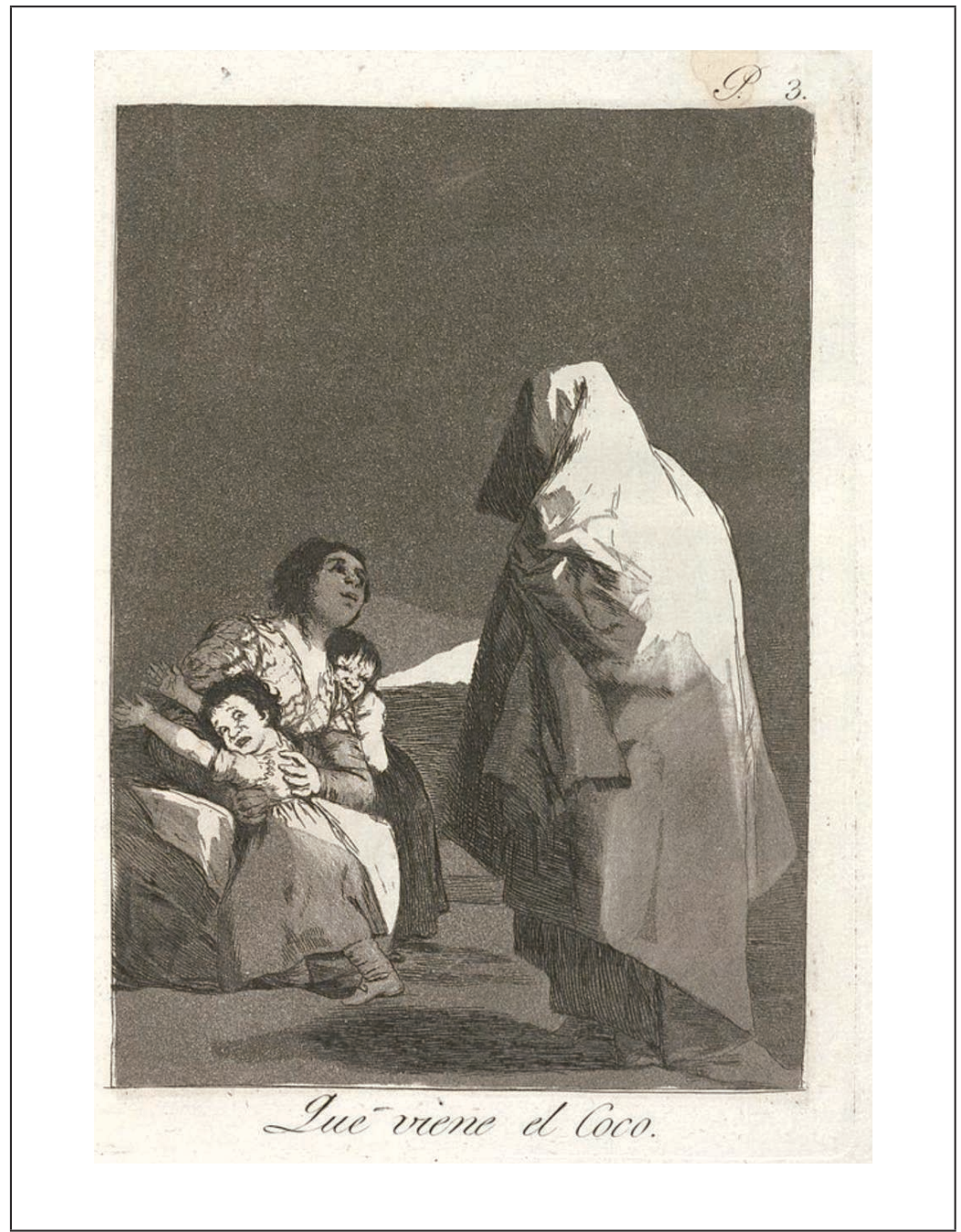

Figure 2: F. Goya Que viene el Coco, 1799, Brooklyn Museum

\section{Le chat, la chienne, la barbe et l'épouvantail}

Selon le TLFi, le mot Croquemitaine serait « composé de la forme verbale croque (croquer*) et d'un $2^{e}$ terme d'origine incertaine. Il pourrait s'agir d'une composition comparable à grippeminaud, où mitaine* désignerait alors le chat, compagnon du diable à qui l'on enjoindrait de manger les enfants (GUIR. Étymol., pp. 30-31)».

A partir de cette définition, nous mettons en évidence la dimension iconique de la motivation du signe. Selon Contini 2009, celle qui : « définit un référent par rapport à des 
traits saillants : le nom d'un animal, par exemple, peut renvoyer à l'une de ses caractéristiques physiques, à son activité, à sa relation à l'homme ou à l'univers culturel des hommes à un moment de leur histoire».

Nous avons déjà eu l'occasion de témoigner de sa productivité (Poli 2015).

\subsection{U ghjattumammò ${ }^{3}$ et Dentilonga}

Nous retrouvons ici l'évocation du chat : ghjattu, gattu ou misgiu, selon les localités.

Cette créature à la fois « chat » et « grand-mère » mammona convoque aussitôt l'image ambivalente de la vieille femme mêlée à la méfiance que suscite le chat, à qui l'on prête des pouvoirs surnaturels.

Pourtant, sous l'appellation gatto mammone, la même créature peuple le folklore italien mais rien ne semble la relier à la notion de «grand-mère», dénommée par ailleurs nonna en Italie. Le dictionnaire Garzanti rappelle : "mostro delle favole, che si menziona per far paura ai bambini ». Il s'agit bien du croquemitaine. En revanche mammone renverrait à l'arabe maimūn « singe », par analogie avec ses déplacements.

Avec ghjattumammò, nous sommes manifestement en présence d'une remotivation. Mammone et sa forme apocopée mammò auraient pris ici le sens de « grand-mère », l'origine du mot étant manifestement désapprise ${ }^{4}$ depuis longtemps. Il s'agirait d'un cas d'attraction paronymique comme il en existe bien d'autres en langue corse. Nous avions consacré un développement à ce phénomène, appliqué aux phytonymes et aux zoonymes, dans notre étude de doctorat (Poli 2001).

Quant au chat, il est régulièrement associé aux forces du Mal : diable et sorciers. Objet de culte dans L'Egypte ancienne, le chat a toujours plutôt suscité la méfiance en Occident. Il est au centre de toutes les croyances. Animal de mauvaise augure, surtout quand il est noir, on lui prête sept vies (ou sept âmes). Et dans l'imaginaire populaire, on le tenait pour un auxiliaire des sorcières. On dit même que le chat est la forme la plus commune que les sorcières prennent quand elles courent la campagne la nuit, pour se livrer à leurs méfaits.

On peut comprendre qu'il soit ainsi étroitement lié à l'image sinistre du croquemitaine.

Linformatrice d'Ochjatana, Madame Leoni, propose une hiérarchisation intéressante des monstres qui peuplaient son enfance (1), u ghjattumammò représentant le summum de l'effroi :

(1) Peghju ch'à Cagna Milana. Cagna Milana era quantunque più abburdevule ! U ghjattumammò, sischia l'osse à e ghjente !

Pire que Cagna Milana5. Cagna Milana était quand même plus abordable! Le ghjattumammò nous épouvantait ${ }^{6}$ !

3. Le dictionnaire U Muntese mentionne : «gattumammone n.m. Croquemitaine »

4. Actuellement, l'entrée « croquemitaine » de la BDLC renvoie à deux formes : ghjattumommò à Guagnu et mommò à Eccica Suarella. (d'après http://bdlc.univ-corse.fr, consulté le 2 mai 2015)

5. Voir infra

6. Plus exactement : « glaçait les os». 
Elle commente également le stratagème dissuasif mis en place par ses parents (2):

(2) Ci tinianu, pè fà frenu, cù u ghjattumammò perchè ùn surtiamu micca tardi fora. Zitelli ùn si ne vidia! Dopu elli ùn ci cridianu più, grandi ch'elli eranu! Ma noi altri zitelli obediamu è stavamu à sente chì u ghjattumammò... altrimente u truvavamu.

Ils nous retenaient avec $u$ ghjattumammò pour qu'on ne sorte pas le soir. On ne voyait pas d'enfants (dehors)! Ensuite, devenus grands, ils n'y croyaient plus! Mais, petits, nous obéissions et écoutions nos parents, sinon on le croisait.

La désignation ghjattumammò est relativement répandue en Corse. Mommò est attesté à Ajaccio et la forme ghjattumiò à Olmi Cappella.

Le fait que gatto mammone semble très commun dans le vocabulaire italien nous encourage à penser que là est son origine.

À contrario, le croquemitaine de Novella Dentilonga semble très localisé.

On imagine aisément la terreur des enfants lorsqu'on évoquait la créature pourvue de longues longhe dents denti.

Ici encore, a Dentilonga permettait de maintenir les enfants chez eux le soir.

Madame Karceles Giudicelli en témoigne (3):

(3) «Ah quessa era Grand-mère, quandu vuliamu sorte o ch'o vuliamu andà in qualchì locu. Dicia : «nò, ùn andate perchè ci hè a Dentilonga chì si piatta quassù ind'è u campanile ! Ùn passate micca custì.» (...) Vulianu chè no stessimu in casa. (...) Surtout a sera quand'è no surtiamu, u ghjornu eramu à a scola!

Era una fola!» Mammò $^{7}$, lorsqu'on voulait sortir, disait : « Attention! Il y a Dentilonga cachée là-haut dans le clocher! Ne passez pas par là ». Elle voulait qu'on reste à la maison. Surtout le soir, dans la journée nous étions à l'école. C'était une histoire!

\subsection{Cagna Milana ${ }^{8}$, Barbarone et Franca Pellella}

Cagna Milana et Barbarone ont la particularité d'être liées à l'acte alimentaire au moment du carnaval précédant le Carême ou aux fêtes de Noël.

Le récit de Cagna Milana est situé à Costa, toute petite commune de l'intérieur, à 1,5 kilomètre d'Ochjatana cité plus haut. Ici, le croquemitaine est brandi pour inciter les enfants à manger.

Le signifié cagna nom corse de la « chienne », pourrait tout autant qualifier une femme

7. «Grand-mère »

8. Le dictionnaire U Muntese (réed. 2014) mentionne : « gattumammone n.m Croquemitaine » 
sensuelle et sans moralité. Les propriétés malsaines rappellent sans mal les caractéristiques de la sorcière.

On aurait retrouvé la trace au XVIIe siècle, en Italie (en Ligurie, à San Pietro di Novella précisément), à San Pietro di Novella précisément, de la condamnation d'une femme nommée Cagna corsa $a^{9}$ possiblement originaire de Corse, accusée et brûlée pour sorcellerie.

Monsieur Mancini, notre témoin, dépeint le rôle de Cagna Milana (4) et situe les faits dans le temps:

(4) «Era vicinu à Quaresima è ci vulia à manghjà assai. È c'era una vechja chì ci cuntava chì, s'ellu ùn si manghjava micca, c'era una strega, Cagna Milana, a chjamavanu. U so travagliu era quessu custì. I zitelli, s'elli ùn avianu micca manghjatu abbastanza i si purtava. »

C'était avant le Carême et il fallait beaucoup manger. Une vieille racontait que s'il on ne mangeait pas, on appelait la sorcière Cagna Milana. Son travail était d'emporter les enfants qui n’avaient pas assez mangé. »

Comment ne pas y voir de similitude avec le mythe de la Vieille de Yennayer présentée par Paquès 1975 lorsqu'elle relate: "Ces cérémonies entrainent l'usage de nourritures particulières sèches ou humides selon le caractère de la fête. Il s'y ajoute l'idée du gonflement du ventre : la vieille iïennayer ouvre le ventre des enfants qui ont trop mangé et chatouille les pieds de ceux qui ne l'ont pas fait suffisamment. »

Et sans toutefois qu'il y fasse état d'une quelconque créature, ne pourrait-on pas faire le lien avec les propos de Videgain 2005 ? Il précise qu' « En Navarre et Alava (près de Pampelune et Vitoria) on désigne par le castillan 'la vieja', le temps de Carême. Cette notion de zahar liée au cycle de l'année est certainement à explorer plus précisément... ». On assiste simplement à une représentation anthropomorphique des saisons, une personnification de l'année qui touche à sa fin.

Barbarone, personnage redouté dans le village de Speloncatu, où elle sévit (5) rejoint la même thématique:

(5) « Barbarone... era una vechja chì girava nant'à i tetti è quandu eramu gattivi ci punia, surtuttu di Natale, ind'è i scarpi c'era un suppulu di carta è u carbone, spentu!

Barbarone était une vieille qui déambulait sur les toits et quand nous étions méchants, elle nous punissait, surtout à Noël, on retrouvait dans les chaussures un peu de papier et du charbon, éteint!

9. «Cattarina, detta la Cagna corsa, proveniente forse dalla Corsica. » (Cattarina dite la Cagna corsa, peut-être originaire de Corse »). Révélé, à l'origine, dans le périodique Il mare du canton de Tigullio, en juin 1909. 
Madame Gaubert-Grimaldi précise ses souvenirs (6) :

(6) «Una sera, ùn c'era micca lume, eramu à a lampana à petrollu, (...) Eramu tutti à mezu à u vighjone è d'un colpu quandu ùn ci pinsava micca mi casche à i pedi un pacchettu. Uh Diu ! Chì hè ? Laghju apertu è c'eranu dui canistrelli è un'aranciu. «Quale hè ? » "Hè Barbarone chì hè passata parchè sì stata brava ». Ùn aghju micca vistu da induv'ellu hè falatu ! (...) Ma ci credia talmente ! » Un soir, il n'y avait pas de lumière et nous étions éclairés à la lampe à pétrole. Nous étions tous à la veillée et d'un coup, quand je ne m'y attendais pas, un paquet tombe à mes pieds. Mon dieu! Qu'est-ce que c'est? Je l'ai ouvert. Il contenait deux canistrelli ${ }^{10}$ et une orange. Qui est-ce? C'est Barbarone qui est passée car tu as été gentille. Je n'ai pas vu d'où il est tombé. Mais j'y croyais tellement !

On note ici l'ambivalence du personnage soulignée par Belmont 2004 : « D’autres êtres fantastiques anthropomorphes peuvent se grouper sous un type particulier; ce sont des personnages bienveillants envers les enfants qui, parfois, sont invoqués pour leur faire peur ou qui se dédoublent en personnage bienveillant et personnage terrifiant. Sous ce type, on trouvera tous les êtres surnaturels qui apportent des friandises et des jouets durant l'époque qui s'étend de la Saint-Nicolas (6 décembre) au Jour des Rois (6 janvier). »

Nos deux témoignages rappellent la légende de la Befana du folklore italien, dont le nom évoque l'Epiphanie et d'autres figures bien connues des contes populaires.

On pense bien entendu, à la sorcière d'Hänsel et Gretel ${ }^{11}$ qui au premier abord leur apparaît comme une charmante grand-mère, leur offrant : " du lait et de l'omelette au sucre, des pommes et des noix » dans sa maison de pain d'épices. Or, ce n'est qu'une ruse pour attirer les enfants et les manger, car la sorcière est aussi un peu ogresse... tout comme Baba-Yaga, la grand-mère sorcière des contes russes populaires, qui vit dans la forêt et enlève les enfants pour les dévorer.

Et si ces femmes malfaisantes sont communément appelées sorcières, elles répondent également à la définition du croquemitaine, puisque leur évocation est destinée à provoquer l'obéissance des plus jeunes.

Le personnage de Barbarone fait écho au Barbau/Barbaou que l'on peut trouver en Bretagne. La forme Barbarone semble motivée par la «barbe »barba ${ }^{12}$; l'ajout du suffixe augmentatif -one exprime en même temps une certaine forme d'admiration, voire de crainte. Limage de l'homme poilu, l'ome pelut de l'Aude mentionné par Sébillot 1905 est commune parmi les dénominations du croquemitaine.

N'omettons pas de considérer également les nombreux travaux qui ont mis en évidence la possibilité qu'ont les productions sonores d'évoquer des images ou des sensations. Citons Contini 2009, lorsqu'il indique : «Symboliquement le sentiment de peur, associé au noir et à

10. Spécialité pâtissière : gâteau sec.

11. Contes pour les enfants et les parents, J. \& W Grimm, 1812-1829.

12. Voir infra, A Capra Barbantana. 
l'au-delà, a pu être évoqué par l'emploi de réalisations phonétiques marquées par le trait grave caractérisant à la fois les voyelles vélaires, sombres, et les consonnes labiales ».

Dans les appellations Barbarone ou Mommò, on devine : «l'impression sonore des voix attribuées aux êtres fantastiques (...) suscitant la crainte et supposés entretenir des relations avec l'au-delà » (Contini 2009).

Franca Pellella, sorte d'ogresse, sévissait au-dessus d'Ile Rousse à Monticellu.

Sa désignation [fr'anka bel'ela] demeure assez opaque. Il semble toutefois clair que, comme le terme Francalossu — l'une des désignations de l'« épouvantail » en corse — elle soit formée par composition du verbe francà et d'un substantif certainement altéré au fil du temps. Il pourrait s'agir d'un dérivé de pella $a^{13}$ « peau ». A pellella serait celle qui épargne franca la peau. D'ailleurs, la formulette rattachée à son évocation n'est-elle pas?

« Franca Pellella, si manghja l'osse è si lascia a pelle »

Franca Pellella, elle mange les os et elle laisse la peau

Sans cette formulette destinée à entretenir l'effroi suscité par l'apparition de l'ogresse, on aurait pu penser à une femme habillée de peaux, tels les pellicciari du cortège de Carnaval ${ }^{14}$.

Contée par Monsieur Graziani, rapportant les souvenirs de son oncle conteur à la veillée, il établit une échelle de la crainte (7) sur laquelle Franca Pellella figure en bonne place:

(7) Ci n'era una chì facia a paura, era a Vechja Cannuta ${ }^{15}$, ùn m'arricordu più tantu ma c'era Franca Pellella, era peghju!

Celle qui faisait peur, c'était A Vechja Cannuta, je ne m'en souviens plus trop, mais il y avait Franca Pellella, elle était pire qu'elle!

Il précise (8) comment elle s’y prenait pour terrifier la population :

(8) Franca Pellela era una streia, stava quindi ind'è a Culumbaghja. Tutte e sere vinia ind'è u paese. (...) Facia u giru di e case è quand'ella sintia ch'ellu c'era zitelli micca bravi, apria a porta, si pigliava u zitellu è u si ne purtava.

Franca Pellella était une sorcière qui habitait A Culumbaghja ${ }^{16}$. Elle venait tous les soirs, faisait le tour des maisons et quand elle entendait des enfants désobéissants, elle ouvrait la porte et elle les emportait.

13. Pelle dans le parler balanin

14. Cités en marge du récit de Barbarone, à Speloncatu.

15. La « vieille à la canne».

16. Toponyme commun, construit sur culombu « pigeon » + suffixe aghju (ARIUM Latin) = repère de pigeons. 


\section{Le mythe de la « vieille»}

Si nous avions recueilli ni description ni détails sur l'apparition de ces personnages, nous aurions pu penser qu'il s'agissait d'êtres verbaux « ces êtres fantastiques dont la seule caractéristique semble être le nom, sur lequel repose toute la vertu d'effroi qu'ils suscitent » comme l'indique Belmont 2004.

Leurs portraits demeurent flous. Ils existent uniquement dans la mémoire des locuteurs les plus anciens.

A quoi ressemblent des monstres? Avant tout, à de vieilles femmes inquiétantes.

\subsection{Une femme-chef}

Pierre-Marie Mancini précise l'idée (9) qu'il s'en faisait, enfant :

(9). « Cagna Milana facia u giru di i paesi (...) era una strega affiancata da i so maghi, chì eranu un pocu a so armata, è girava u paese incù e botte, è intria ind'è e case à piglià i zitelli. Ùn si sapia micca da induv'ella vinia. À a longa, noi eramu insischiti! »

Cagna Milana faisait le tour des villages. C'était une sorcière épaulée par des sorciers qui constituaient son armée en quelque sorte; elle traînait dans le village avec ses tonneaux et entrait dans les maisons pour y prendre les enfants. On ne savait pas d'où elle venait. Et à la longue, ça nous terrifiait !

Le tonneau joue le rôle du sac ou de la hotte, attribut indispensable de celle/celui qui enlève les enfants.

Habituellement solitaire, elle est ici accompagnée par d'autres personnages mystérieux.

Il rapporte également la fois où la créature a été vaincue (10) in extremis, preuve de sa relative vulnérabilité :

(10). "Ci cuntava ancu chì era ghjunta ind'è ella è chì, ancu di grazia chì u babbu era custì ! È chì li hà sciaccatu una bastunata è si n'hè andata. Ma chì altrimente sarebbe stata pigliata da Cagna Milana! ».

Elle nous racontait aussi qu'elle était venue chez elle et que par chance son père était là. Il l'avait rossée et elle était partie. Sinon, elle aurait été enlevée par Cagna Milana!

\subsection{Des acrobates isolées}

Certaines familles de Speloncatu suggéraient (11) une vieille dame déambulant sur les toits :

(11). « Allora quessa a mi riprisentava cum'è una cugina carnale di a mo mammò chì stava sopra, a chjamavanu Zia Mariannè. Stava sola è era un suppulu speciale. Allora a mi riprisentava cum'è ella : incù e so robbe longue, u so caraccò... u mandile... cusì, ma chì passava nant'à i tetti. (...)» 
Alors, celle-là je l'imaginais semblable à une cousine germaine de ma grandmère qui vivait au-dessus de chez nous : Zia Mariannè. Elle vivait seule, elle était un peu spéciale. Je me la représentais comme elle : avec des robes longues, un corsage, le fichu... comme ça, mais qui marchait sur les toits

Le croquemitaine qui terrorisait Monticellu était aussi une sorcière, particulièrement vilaine (12) qui ne tombait jamais (13).

(12). «Era assai goffa, avia un nasu goffu, una bocca tamanta, l'arechje fora, cumu un lupu. Avia u mandile in capu, strettu bè è vistuta à la longa. Incù un saccu tamantu è mittia e criature indrentu ».

Elle était très moche, avait un vilain nez et une très grande bouche, les oreilles proéminentes comme un loup. Elle avait un fichu sur la tête, bien serré et longuement vêtue. Avec un sac énorme dans lequel elle mettait les enfants.

(13). «Eranu sopr'à tuttu a sera ch'elle vinianu, a notte. Ùn c'era micca lume ind'è e chjasse... Elle ùn cascavanu mai ! Ùn eranu micca cum'è noi, ùn avianu micca a paura elle ; eranu streie, capite!»

Elles sortaient surtout la nuit. Les chemins n'étaient pas éclairés... Elles ne tombaient jamais. Elles n'étaient pas comme nous, elles n'avaient pas peur elles, c'était des sorcières !

Il s'agit toujours de femmes malfaisantes, vivant seules, retirées (tout comme Dentilonga retranchée dans un clocher), sortant uniquement la nuit et dotées de pouvoirs prodigieux.

\subsection{Des femmes fantasques}

En cela, elles rappellent les véritables streie, ces femmes bien réelles qui pendant la journée vaquaient à leurs occupations ordinaires et le soir venu, se travestissaient pour faire peur à la population.

Cet usage était très répandu en Corse. Notre corpus le relate largement. Canzunella, Luciona et A Maestra Barbuta en font partie.

La nuit venue, elles se dissimulent sous des vêtements occultants, fasciate ou ingutuppate enveloppées, accapparunate encapuchonnées, manicuperte les mains cachées. Et lorsqu'elles ne guettent pas les passants pour les effrayer au détour d'une ruelle, elles déambulent dans le maquis et la forêt parcourant des distances improbables, sans crainte des bêtes sauvages.

Madame Massimi évoque (14) les apparitions de Canzunella, la sorcière de Calenzana :

(14). «Allora sta Canzunella, ella ghjucava nant'à st'affare custì. Certe volte si mittia i stracci, un linzolu biancu. È ind'è a nuttata, iscia, è s'impustava ind'è e carrughje cusì. Sinnò era una donna cum'è tutte e donne! Andava à fà e bucate cù a cennare nant'à u fiume, ind'è U Seccu... Di tantu in tantu li pigliava u sciantigliu, s'arrizzava ind'è a nuttata eppò andava à impustassi à a cunfraternita. Vulia fà pinsà ch'ella era una finzione ! » 
Alors, cette Canzunella, jouait à ces choses-là. De temps à autres, elle se couvrait de haillons, d'un drap blanc. Et la nuit venue, elle sortait et se postait dans les rues comme ça. Sinon, c'était une femme comme les autres! Elle faisait les lessives, au fleuve du Seccu avec la cendre... De temps à autres, elle perdait la tête, se levait la nuit et se mettait en embuscade derrière la confrérie. Elle voulait que l'on croie à un fantôme.

Notre informateur de Palasca mentionne une femme inquiétante (15) dénommée A Maestra Barbuta :

(15). A Maestra Barbuta... què po allora ! Hè una donna incù issa robba negra eppò hà sempre un saccu nant'à a spalla ! Stava in Olmi Cappella, una struitona ! È falava in Lozari : dava i corsi à i zitelli. Francava. C'era una trentina di chilometri ! Facia issu viaghjone. Dopu li anu messu una barba pè fammi paura. Eccu a storia d'issa donna, ma allora vi guasta una zitillina, bah !

La maîtresse barbue, ça alors! C'est une femme habillée de noir, toujours avec un sac sur l'épaule! Elle habitait Olmi Cappella, elle était très instruite! Et elle descendait à Lozari : elle y donnait des cours aux enfants. Elle traversait. Il y avait une trentaine de kilomètres! Elle parcourait ce long trajet. Ensuite, ils lui ont mis une barbe pour me faire peur. Voilà comment une histoire peut vous gâcher votre enfance, eh !

Dans la vallée du Filosorma, Monsieur Simeoni se souvient de Luciona (16):

(16). C'era una vechja, era una strega. A chjamavanu Luciona, l'aghju cunnisciuta bè. Li chjappava a notte, si mittia u longu à u Strettu ${ }^{17}$ è durmia. Ancu i cignali è e volpe avianu a paura di ella E ghjente n'avianu a paura! Ella scalza, vistuta in neru, dicia : « Fila a to strada!». C'était un personnage, hein! Ùn c'era c'à ella chì vivia cusì.

Il y avait une vieille, c'était une sorcière. On l'appelait Luciona, je l'ai bien connue. Lorsqu'elle se faisait surprendre par la nuit, elle s'allongeait le long du Strettu et elle dormait. Les sangliers et les renards en avaient peur. Les gens en avaient peur! Elle, pieds-nus, vêtue de noir, disait : «Passe ton chemin !». Il n'y avait qu'elle qui vivait comme ça.

Notons enfin, que parmi ces êtres inquiétants évoluent également des créatures zoomorphes : a capra barbantana ${ }^{18}$, a Biscia de l'Ostriconi ${ }^{19}$ ou u porcu biancu ${ }^{20}$ de Sant'Antuninu sont autant de garde-fous.

17. Toponyme signifiant un détroit, un passage entre deux cols.

18. Chèvre monstrueuse, véritable tourment pour notre informateur de Palasca.

19. Du nom du fleuve qui l'abritait.Monstre lacustre assimilé à un serpent dévorant les chrétiens lorsqu'ils se rendent à la messe. La légende dit qu'il fut battu par un valeureux guerrier, mort à son tour empoisonné par sa propre épée souillée par le sang de la bête.

20. Âme errante rencontrée sous la forme d'un porc blanc, animal psycopompe au regard de la tradition locale. 


\section{Conclusion}

Depuis des siècles, l'éducation de l'enfant dans les sociétés traditionnelles « se fait par la crainte et l'ironie ${ }^{21} »$.

En Corse, jusqu'à la fin de la première moitié du XXe siècle, à la veillée, on privilégiait e fole chì arricavanu a paura è i stalvatoghji ${ }^{22}$.

Concernant le croquemitaine précisément, les spécialistes semblent divisés quant à la datation de son apparition. Certains font remonter son existence au début du XIX ${ }^{e}$ siècle et estiment qu'il n'était pas connu dans les campagnes, alors que d'autres ${ }^{23}$ se livrent à une caractérisation des « figures du croquemitaine médiéval » à partir de sources écrites...

Néanmoins, selon les régions et les époques, le loup (garou), le Diable, l'ogresse sortes de constantes - ont pu faire office de croquemitaine, parallèlement à des créations locales qui racontent la société dans laquelle elles ont évolué. La nuit, la forêt, l'eau, le repas représentent autant de sujets de dissuasion pour les parents.

Pourtant, les interdits ne sont pas les mêmes partout. Ils diffèrent selon les occupations et les préoccupations des communautés. Et, le recours au personnage comme moyen éducatif est l'apanage de l'oralité.

On a coutume de dire que e fole sont salutaires car aussi effrayants que soient ces récits, l'enfant sait qu'il ne court aucun danger s'il obéit.

Ne dit-on pas qu' « il faut trembler pour grandir»? On nomme le mal et en le nommant, on l'exorcise.

21. Nicole Belmond 2004, en témoigne, s'appuyant sur les thèses de Van Gennep.

22. "Histoires terrifiantes et ridicules" ainsi les qualifie notre informateur d'Aregnu.

23. Berlioz, Alexandre Bidon 1988. 


\section{Bibliographie}

Belmont Nicole, "Comment on fait peur aux enfants », La lettre de l'enfance et de l'adolescence 2/2004 (n० 56), pp. 51-58

Berlioz Jacques, Alexandre-Bidon Danièle, Le masque et la barbe, figures du croquemitaine médiéval, in Le Monde alpin et rhodanien, n²-4, 1998, pp.163-186

Contini Michel, Les phonosymbolismes : continuité d'une motivation primaire?, in Travaux de linguistique, 2009, De Boeck Supérieur, pp. 77-103

Paquès Viviana, Carnaval, fête du mariage et de la mort, in Revues des sciences sociales, $n^{\circ} 4$, 1975

Patera Maria, Comment effrayer les enfants : le cas de Mormô/Mormolukê et du mormolukeion, Kernos, 18 | 2005 [En ligne]

Poli Muriel, Phytonymes et zoonymes corses - Etude de sémantique lexicale et de géolinguistique, Thèse de doctorat, Université de Corse, 2001

Poli Muriel, Quand la polysémie se généralise, le labre, le roitelet et l'euprocte se font 'vieux', Bretagne Linguistique, $\mathrm{n}^{\circ} 19,2015$, pp.27-42

Sébillot Paul, Folk-Lore de la France, La mer et les eaux douces, Librairie orientale \& américaine, 456 pages, 1905 ,

Van Gennep Arnold, Du berceau à la tombe, tome1, volume 1, Paris, Picard, 1943-1946

Videgain Charles, Vieillir en basque et zahar, in Les mots du vieillir, Presses Universitaires Blaise Pascal, pp.23-29, 2005 